\title{
Scaling laws of graphs of 3D protein structures
}

${ }^{1}$ Faculty of Mathematics, Natural Sciences and Information Technologies, University of Primorska,

${ }^{2}$ Department of Biochemistry, Molecular and Structural Biology, Institute Jožef Stefan, Jamova 39, Ljubljana, Slovenia 


\section{Abstract}

The application of graph theory in structural biology offers an alternative means of studying 3D models of large macromolecules, such as proteins. However, basic structural parameters still play an important role in the description of macromolecules. For example, the radius of gyration, which scales with exponent $\sim 0.4$, provides quantitative information about the compactness of the protein structure. In this study, we combine two proven methods, the graph-theoretical and the fundamental scaling laws, to study $3 \mathrm{D}$ protein models.

This study shows that the mean node degree of the protein graphs, which scales with exponent 0.038 , is scale-invariant. In addition, proteins that differ in size have a highly similar node degree distribution, which peaks at node degree 7 , and additionally conforms to the same statistical properties at any scale. Linear regression analysis showed that the graph parameters (radius, diameter and mean eccentricity) can explain up to $90 \%$ of the total radius of gyration variance. Thus, the graph parameters of radius, diameter and mean eccentricity scale with the same exponent as the radius of gyration. The main advantage of graph eccentricity compared to the radius of gyration is that it can be used to analyse the distribution of the central and peripheral amino acids/nodes of the macromolecular structure. The central nodes are hydrophobic amino acids (Val, Leu, Ile, Phe), which tend to be buried, while the peripheral nodes are more hydrophilic residues (Asp, Glu, Lys). Furthermore, it has been shown that the number of central and peripheral nodes is more related to the fold of the protein than to the protein length. 


\section{2}

73

74

\section{Introduction}

Proteins, molecules that serve many critical roles in nature, consist of complex systems of amino acids that have increasingly been modelled as networks over the last decade (1-3). There are many ways to abstract the $3 \mathrm{D}$ protein structure into a graph. We can consider $\mathrm{C} \alpha, \mathrm{C} \beta$ or all heavy atoms to construct an adjacency matrix. A typical method to abstract the protein model into a graph is to consider C $\alpha$ atoms with $7.0 \AA$ cut-off distance (4). We should be aware that some information is lost when the 3D model is abstracted into the graph, although it still captures relevant biochemical properties of the "real" 3D protein model. Once the 3D model of the protein is abstracted into a graph, we have several options to analyse the 3D structure by examining different parameters of the graph. Residue network models have been used to predict catalytic sites (5-8), to study protein dynamics (9), to discover node-amino acids that play crucial roles in protein folding $(10,11)$, to explore allosteric pathways (12-14), and to analyse enzyme domain packing (15). In addition, graph theory has also been successfully used to validate PDB entries (16), to study local errors in protein models, and to discriminate decoys from the native structure $(17,18)$. We should emphasize the importance of validation and quality assessment, since only correct protein structures can answer relevant biological questions (19-22).

However, we cannot expect that all graph parameters used and derived by mathematicians will have practical implications, for example, when studying specific phenomena in structural biology.

However, we should try to find a connection between theoretical graph parameters and biochemical phenomena that can lead to deeper insights.

Despite the obvious usefulness of graph theory for the analysis of protein structures, basic structural parameters still serve an important role in the description of macromolecules. For example, a common parameter used to describe the compactness of protein models is the radius of gyration (23-25). The radius of gyration of a macromolecule describes the distribution of atoms around the centre of mass. Since Flory's theory (26), the scaling law between the radius of gyration and the length of the protein has been studied in detail and used to describe protein folding, and to analyse 
the compactness of protein structures in poor or good solvent conditions. It was found that the radius of gyration of globular protein structures, including monomers and oligomers, scales with exponent $\sim 0.4$ (27). The radius of gyration can be used as a constraint when building protein models or performing molecular dynamics simulations. On the other hand, when the 3D model of a protein is not known, the scaling law provides qualitative information about the dimensions of the macromolecule.

104 This study links two well-established approaches, the graph-theoretical and the fundamental scaling 105 laws, to study 3D protein models. In this paper, the PDB is surveyed to study scaling laws of 3D protein structures using a graph-theoretical approach. This research has demonstrated that the mean node degree of the protein graph is nearly scale-invariant. Additionally, the comparison of the node degree distributions of proteins of different sizes exhibits marginal differences. Furthermore, this study shows that the compactness of the protein, which is conventionally calculated by the radius of gyration, can be estimated using graph eccentricity, which also provides insights into central

111 (buried) and peripheral (non-buried) amino acids.

\section{Methods}

\section{Dataset}

115 The Protein Sequence Culling Server (28) was used to obtain the PDB id list for protein structures 116 with the following characteristics: maximum mutual sequence identity of $80 \%$, X-ray resolution 117 cut-off of $3.0 \AA$ and minimum (maximum) chain length of $40(10,000)$ residues. The PDB id list 118 was then used to retrieve 31,571 Biological Assemblies from the Protein Data Bank.

\section{Graph Construction}

121 From each of the 31,571 3D Biological Assemblies, the graph was constructed and analysed. C $\alpha$ 
123 between a pair of residues was less than (or equal to) $7 \AA$. It follows that the number of nodes was

124 equal to the number of residues ( $\mathrm{C} \alpha$ atoms) in the protein. Ligands, water molecules, and other

125 hetero-compounds were discarded during graph construction. Thus, if a protein has $n$ residues, then

126 a protein graph $G=G(V, E)$ consists of a set of vertices (nodes) $V=v_{1} v_{2}, \ldots v_{n}$ and a set of edges $\mathrm{E}=\mathrm{e}_{1}, \mathrm{e}_{2}, \ldots \mathrm{e}_{\mathrm{m}}$.

\section{Graph parameters}

130 The mean node degree (MND) of a graph G is expressed with the ratio

$$
d(G)=\frac{2 e(G)}{N(G)}
$$

where $\mathrm{e}(\mathrm{G})$ represents the total number of edges in a graph $\mathrm{G}$, and $\mathrm{N}(\mathrm{G})$ is the number of nodes in a graph G.

134 The eccentricity is a node centrality index defined as the maximum distance between a vertex to all 135 other vertices. Thus, the vertex's eccentricity is the maximal shortest path between the vertex and all other vertices. Mean eccentricity is expressed as an average value of eccentricities of all vertices of G. The radius of the graph is defined as a minimum eccentricity among all vertices in the graph. Meanwhile, the diameter is defined as maximum eccentricity among all vertices in the graph. The center of a graph or central node has eccentricity equal to the radius. A vertex is said to be a

140 peripheral node if its eccentricity is equal to the diameter.

141 Next, R script (igraph package) was used to calculate mean node degree, eccentricity, mean 142 eccentricity, radius and diameter: 
149 Radius of gyration

150 Considering atoms as points in a three-dimensional space, the radius of gyration is defined as

$$
\mathrm{R}_{\mathrm{gyr}}=\sqrt{\frac{\sum \mathrm{m}_{\mathrm{i}} \mathrm{r}_{\mathrm{i}}^{2}}{\mathrm{M}}}
$$

152 where $\mathrm{M}$ is the total mass of the molecule, and $\mathrm{m}_{\mathrm{i}}$ is the mass of the $\mathrm{i}$-th atom with distance $\mathrm{r}_{\mathrm{i}}$ from

153 the centre of mass. Radius of gyration was calculated using the rgyr function, which is part of the

154 Bio3D R package (29).

\section{Solvent accessibility of residues}

157 Secondary structure (total solvent-accessible area of proteins) was assigned according to the method 158 of Kabsch and Sander $(30,31)$. The solvent accessible area data for protein residues were taken from 159 the work of Tien and co-workers (32).

160

161 Table 1: Solvent accessibility of residues

\begin{tabular}{|c|c|}
\hline Residue & $\begin{array}{c}\text { Solvent } \\
\text { accessible } \\
\text { area }\left(\AA^{2}\right)\end{array}$ \\
\hline Alanine & 121 \\
\hline Arginine & 265 \\
\hline Asparagine & 187 \\
\hline Aspartate & 187 \\
\hline Cysteine & 148 \\
\hline Glutamate & 214 \\
\hline Glutamine & 214 \\
\hline Glycine & 97 \\
\hline Histidine & 216 \\
\hline Isoleucine & 195 \\
\hline Leucine & 191 \\
\hline Lysine & 230 \\
\hline Methionine & 203 \\
\hline Phenylalanine & 228 \\
\hline Proline & 154 \\
\hline Serine & 143 \\
\hline Threonine & 163 \\
\hline Tryptophan & 264 \\
\hline
\end{tabular}


162

163

\section{4}

165

166

167

168

169

170

171

172

173

174

175

176

177

178

179

180

181

182

\section{Results and Discussion}

\begin{tabular}{|c|c|}
\hline Tyrosine & 255 \\
\hline Valine & 165 \\
\hline & \\
\hline Average & 192 \\
\hline
\end{tabular}

\section{Node degree-nearly scale-invariant}

One of the fundamental global graph parameters in graph theory is the mean node degree (MND).

The mean node degree shows how many edges each node has on average. In previous work,

Pražnikar and co-workers (33) have shown that protein models that deviate from the expected MND

by approximately two standard deviations or more are likely to be incorrect. Furthermore, the

scaling exponent calculated in the mentioned study is close to zero and indicates that the mean node degree is nearly scale-invariant.

In this study, a large non-redundant database of biological units $(31,571)$ was used, rather than

crystal asymmetric units. We can see in Figure 1A that MND is not strongly dependent on protein

size and that the distribution is rather narrow. Upon closer examination, however, the value

determined in our study (0.038) differs slightly from the value (0.024) determined in previous

study. The reason for the different scaling exponents is probably that the datasets are not the same.

An analysis performed on two large but different datasets shows that MND is nearly scale-invariant,

i.e., the scaling exponent is close to zero (0.024 and 0.038). Thus, MND is not strongly dependent

on protein length, and it can be concluded that the number of edges in the protein graph is linearly

related to the number of nodes (amino acids).

183 Fig 1. (A) Scaling exponent of mean node degree of protein graphs versus the number of residues.

184 (B) Probability of node degree of protein graphs for three size bins. The first size bin (black line) 
encompasses protein structures with length between 100 and 200 residues, the second size bin (blue line) encompasses protein structures with length between 500 and 600 residues, and the third size bin encompasses structures with length between 900 and 1000 residues.

We could expect that larger proteins would have a higher average node degree because of a higher number of core residues and a relatively lower number of surface residues, which are supposed to

191 have lower numbers of edges. Thus, to further analyse the node degree of protein nodes-residues, we calculated the node degree distribution for three different size bins. The first size bin contains all protein structures from the database, which have lengths between 100 and 200 residues, in the second size bin are proteins with lengths between 500 and 600 residues, and in the third size bin are structures with lengths between 900 and 1,000 residues. Figure 1B shows that all three distributions are very similar and that there is a peak at 7 node degrees. The comparison of all three peak values shows that the first size bin, which contains the smallest proteins, has the highest probability density value. The lowest probability density value at 7 node degree is seen for the third size bin, which contains the largest proteins among all three selected size bins. A closer look at the left (degree 2) and right (degree 14) tail of the distributions shows high similarity for all three distributions. The 201 visual comparison shows the most significant differences on the left and right sides of the peak. The 202 differences can be observed at values of approximately 5 to 9 node degrees. It can be seen that the 203 first size bin has a higher probability at 3 to 6 node degrees as compared to size bins two and three. 204 The order is somehow reversed on the right side of the displayed distribution. For node degrees 8, 9 205 and 10, size bin three exhibits higher values compared with size bins two and three.

206 This analysis shows that despite the different sizes of the proteins, they have a very similar node 207 degree distribution, which peaks at node degree 7. A simple way to explain the presented results is 208 that buried residues in small or large proteins form approximately the same number of links. This is 209 a direct consequence of the fact that the amino acids are physical objects and cannot be arbitrarily 
210 close to each other. The marginal difference in node degree distributions, a slight shift to higher

211 node degrees, explains the low positive scaling exponent (0.038), which is nearly scale-invariant.

213 Protein graph eccentricity: an alternative method for analysis of

\section{4 radius of gyration}

215 It is easy to ask a question: radius of gyration and radius as a graph parameter have a common

216 name, but do they follow the same power law? To answer this question, linear regression analysis

217 and scaling exponent were calculated for three graph parameters: radius, diameter and mean

218 eccentricity. The radius-graph parameter is defined as minimum eccentricity, whereas the

219 eccentricity of the graph is defined as the maximum distance between one node and all other nodes.

220 Notice that the diameter is defined as maximum eccentricity.

221 Figure 2A shows the scaling exponent of a radius of gyration for 31,571 selected protein structures.

222 The non-linear fitting function can be written as

$223 \mathrm{R}_{\mathrm{gyr}}=\mathrm{R}_{0} \mathrm{~N}^{v}$

224 where $\mathrm{R}_{\mathrm{gyr}}$ is the radius of gyration, $\mathrm{R}_{0}$ is the pre-factor and $v$ is a scaling exponent. The pre-factor

$225 \mathrm{R}_{0}$ can be obtained experimentally and used as a restrained value during non-linear fitting (34-36).

226 Thus, when restrained fitting was performed, the pre-factor $\left(\mathrm{R}_{0}=2 \AA\right)$ was fixed. We can see that in

227 the case of restrained fitting, the scaling exponent is 0.405 , which is consistent with other studies.

228 When fitted without restraint, the exponent is lower (0.351). Both values are within the range

229 reported by other studies $(25,27,34,37-39)$.

231 Fig 2. Log-log plots of the radius of gyration (A), radius (B), diameter (C), and mean eccentricity

232 (D) versus the number of residues. The solid red line is generated by fitting without restraint; the

233 blue line is produced by restrained fitting. The legend shows unrestrained scaling factors in red. The 234 restrained scaling factors and corresponding pre-factors are in blue. 
Figure $2 \mathrm{~B}, \mathrm{C}$, and $\mathrm{D}$ show radius, diameter, and mean eccentricity plotted against protein length.

237 Similar to the analysis of the radius of gyration, the power exponent was fitted with and without 238 restraint. The pre-factor for restrained fitting was derived from linear regression analysis, as shown

239 in Figure 3. The linear regression analysis between the radius of gyration and graph parameters reveals that $R^{2}$ is close to 0.90 for all three cases (Fig. 3). The highest $R^{2}(0.91)$ is observed between mean eccentricity and radius of gyration (Fig. 3C). The reason for this is probably that the values of radius and diameter are discrete, while mean eccentricity values are not discrete. For example, the radius can be 7 or 8 , but cannot be a real number between 7 and 8 . Mean eccentricity is just a mean value of all shortest paths to any nodes. It is seen that the distribution of mean eccentricity is smoother in comparison to the discrete values of radius and diameter on the y-axis.

Fig 3. Scatter plot of graph parameters: radius (A), diameter (B), and mean eccentricity (C) versus radius of gyration. The legend shows the regression coefficient, R-squared value, and $\mathrm{p}$-value.

If we use pre-factor $\mathrm{R}_{0}$ of a radius of gyration, which was obtained from experimental data, then we can calculate the pre-factors for radius, diameter, and mean eccentricity using the slope $k$ from a regression analysis. The steepness of the linear regression model between $\mathrm{R}_{\mathrm{gyr}}$ and radius was 0.42 $\AA^{-1}, 0.77 \AA^{-1}$ between $\mathrm{R}_{\mathrm{gyr}}$ and diameter, and $0.59 \AA^{-1}$ between $\mathrm{R}_{\mathrm{gyr}}$ and mean eccentricity (see Fig. $3 \mathrm{~A}, \mathrm{~B}$ and $\mathrm{C}$ ). Using pre-factor $\mathrm{R}_{0}$ and the steepness of linear fit $k$, the pre-factors for radius, diameter and mean eccentricity can be calculated using the next expression:

257 where $\mathrm{R}_{\mathrm{x}}$ is a new calculated pre-factor, $\mathrm{R}_{0}$ is the pre-factor of radius of gyration and $k$ is the steepness of the linear fit. In Figure 2B, C and D are shown calculated pre-factors for radius $(2.0 \AA$ $\left.0.42 \AA^{-1}=0.84\right)$, diameter $\left(2.0 \AA 0.77 \AA^{-1}=1.54\right)$ and mean eccentricity $\left(2.0 \AA 0.59 \AA^{-1}=1.18\right)$.

260 We can see that the restrained scaling exponent is higher than the non-restrained scaling exponent 261 for all three cases. Furthermore, it is observed that restrained graph parameters all have very similar 
scaling exponents $(\sim 0.395)$ which are very close to the scaling exponent of the radius of gyration

$263(0.405)$

264 Thus, this study shows that the radius of gyration, which is calculated from the atomic coordinates

265 and radius of graph follow the same scaling exponent. From this we can conclude that when

266 analysing 3D models of macromolecules using a graph-theoretical theory approach, the eccentricity

267 of the graph can be used to estimate the radius of gyration. Thus, graph parameter eccentricity

268 allows us to investigate whether the 3D protein model deviates from the expected value of the

269 radius of gyration and to obtain information about the compactness of the structure. Furthermore,

270 when a scientist builds a model, and the model is still in an early phase, e.g., as an alanine chain,

271 then the $\mathrm{C} \alpha$ only model, which can be represented as a graph, contains enough information to

272 estimate the radius of gyration.

\section{Central and peripheral nodes}

In the previous section, the relation between the radius of gyration and graph eccentricity was introduced. When exploring graph eccentricity, it is ubiquitous to examine which nodes-amino acids are central (close to every other node) and peripheral (distant from every other node). This kind of analysis has some common points with analysis of solvent-exposed residues, which is the protein; meanwhile, residues exposed to the solvent represent the outer part of the protein in 3D 281 space (40). The molecular mass of a protein is related with the total solvent exposed surface using 282 the next expression:

$A_{\text {monomer }}=4.44 M^{0.770}$,

where $\mathrm{M}$ is the molecular mass of the protein (41). Similarly, we can introduce the relation between protein length and total solvent-exposed surface. Given that the average amino acid has a total solvent exposed area of $192 \AA^{2}$ (32), we can use this value as a restraint during data fitting. Figure 4 shows the protein length against the total exposed area. The scaling exponent of the fitted curve is 
0.772 , almost the same as the exponent in equation 2 . This result was somehow expected because molecular mass correlates with sequence length.

Fig 4. Dependence of total solvent accessible area on the number of residues in proteins. The legend shows the scaling exponent and pre-factor $\left(\mathrm{A}_{0}\right)$, which was used during restrained fitting.

However, a closer examination of the relationship between the number of central (peripheral) nodes and protein length demonstrated that the numbers of central and peripheral residues are not related to the protein size (Figure 5A, B). To further support this finding, the comparison between the radius of gyration, mean eccentricity, and numbers of central and peripheral residues for nine different size bins was made. We can see (Figure 6) that the mean eccentricity and radius of gyration increase according to the length of the protein. Note that the scaling exponent for both mentioned parameters is approximately 0.4 (Figure 2). On the other hand, central and peripheral box plots do not show such a positive trend (Figure 6C, D). It follows that the numbers of peripheral and central residues are not related to the protein size.

Fig 5. Distribution of central/peripheral residues against the size of proteins (number of residues).

Fig 6. Boxplot of the radius of gyration (A), mean eccentricity (B), number of peripheral nodes (C), and number of central nodes (D) for ten different size bins.

Next, we examine the case with different central to peripheral node ratio, which is shown as a graph

310 and ribbon representation of two protein structures (Figure $7 \mathrm{~A}, \mathrm{~B}, \mathrm{D}$, and C). The PDB id: 1pq7

311 structure has a low number of central nodes (4), but a high number of peripheral nodes (47): see

312 Figures 7A and B. The situation is somehow reversed for the PDB id: $3 w v j$ structure, which has a 313 higher number of central nodes (34) and lower number of peripheral nodes (2): see Figure 7D, E. 
314 This case shows that the numbers of central and peripheral nodes depend on the protein fold rather

315 than on the length of the protein chain. Furthermore, we can draw parallels between almost-

316 peripheral (42), self-centred (43), and protein graphs. Figure 7E shows the wheel, a graph that is

317 almost-peripheral, containing only one central node and 6 peripheral nodes. Meanwhile, the graph

318 shown in Figure 7F is an almost self-centred graph that contains 5 central nodes and 2 peripheral

319 nodes. We could say that the graph abstracted from the PDB id: 3 wvj structure is centred, while

320 peripheral nodes-amino acids dominate the PDB id: 1pq7 structure.

Fig 7. Examples of central and peripheral protein graphs. (A) Graph and (B) ribbon presentation of Biological Assembly 1 of PDB entry 1pq7. (D) Graph and (C) ribbon presentation of Biological Assembly 1 of PDB entry 3wvj. (E) Presents an almost peripheral graph, while (F) represents an almost self-centred graph.

Further analysis shows the frequency distribution of central and peripheral amino acids (Figure 8), which are subdivided into three groups: (i) charged, (ii) polar, and (iii) non-polar side chains. It can be seen that amino acids histidine, cysteine, methionine, and tryptophan have the lowest probability values; it follows that they are neither central nor peripheral nodes. Further, we can see that central nodes are hydrophobic amino acids (Val, Leu, Ile, Phe), which tend to be buried, while peripheral nodes are more likely hydrophilic residues (Asp, Glu, Lys) which form hydrogen bonds with solvent.

Fig 8. Probability density of central and peripheral nodes for different types of amino acids.

Thus, when the 3D protein structure is analysed using graph theory, the eccentricity of the graph can be very strong for evaluating the radius of gyration when studying central nodes, which tend to be hydrophobic amino acids, and peripheral nodes, which tend to be hydrophilic amino acids. It is 
340 remarkable that this graph parameter (eccentricity) allowed us to study the compactness $\left(\mathrm{R}_{\mathrm{gyr}}\right)$ of the

341 protein and the arrangement of the residues (buried/not buried), making it versatile and useful for

342 the analysis of 3D macromolecules.

\section{Conclusion}

345 This study showed that the mean node degree of the protein graph is nearly scale-invariant. In other

346 words, a small scaling exponent (0.038) indicates that the mean node degree is scale-free. Scale

347 invariance was further supported by the analysis of node degree distribution, which showed very

348 similar node degree distributions for proteins that differ according to size. This scale invariably

349 offers a valuable tool for validating structures by simply counting the number of edges.

350 Furthermore, an additional comparison between the expected node degree and node degree of a

351 candidate could be used to explore and interpret large deviations. For example, intrinsically

352 disordered proteins are expected to have a considerably lower mean node degree than globular

353 proteins of the same size.

354 The comparison between the mean eccentricity of the graph and radius of gyration revealed a high

$355 \mathrm{R}^{2}$. In other words, the mean eccentricity and radius of gyration follow the same scaling exponent

356 ( 0.4). The eccentricity of the graph, in addition to the estimation of the radius of gyration, also

357 allows us to study the distribution of central (buried) and peripheral amino acids (non-buried). We

358 should be aware that the mean eccentricity alone (or radius of gyration), which is used as a

359 constraint when running molecular dynamics simulations or manually building a model, does not

360 provide the correctness of the protein model. It is also crucial to determine how the amino acids are

361 distributed in real space, and this can be elucidated by studying peripheral and central nodes. Thus,

362 a single graph parameter (eccentricity) can be used to control the compactness of the

363 macromolecule and the distribution of amino acids in 3D space, which makes it a valuable tool for

364 analysing protein models. 


\section{Acknowledgement}

367 This work was supported by Structural Biology grant P1-0048 and Infrastructure programme grant I0-0035-2790, provided by the Slovenian Research Agency.

\section{References}

371 1. Estrada E. Universality in protein residue networks. Biophys J [Internet]. 2010;98(5):890900. Available from: http://dx.doi.org/10.1016/j.bpj.2009.11.017

2. Greene LH. Protein structure networks. Brief Funct Genomics. 2012;11(6):469-78.

3. Vishveshwara S, Brinda K V., Kannan N. Protein Structure: Insights From Graph Theory. J Theor Comput Chem. 2002;01(01):187-211.

4. da Silveira CH, Pires DE V., Minardi RC, Ribeiro C, Veloso CJM, Lopes JCD, et al. Protein cutoff scanning: A comparative analysis of cutoff dependent and cutoff free methods for prospecting contacts in proteins. Proteins Struct Funct Bioinforma [Internet]. 2009 Feb 15 [cited 2017 Mar 15];74(3):727-43. Available from: http://doi.wiley.com/10.1002/prot.22187

5. del Sol A, Fujihashi H, Amoros D, Nussinov R. Residue centrality, functionally important residues, and active site shape: Analysis of enzyme and non-enzyme families. Protein Sci [Internet]. 2006 Sep 28;15(9):2120-8. Available from:

6. Thibert B, Bredesen DE, del Rio G. Improved prediction of critical residues for protein function based on network and phylogenetic analyses. BMC Bioinformatics [Internet]. 2005 Aug 26;6:213. Available from: http://www.ncbi.nlm.nih.gov/pmc/articles/PMC1208857/ [Internet]. 2003 Feb 21;326(3):955-78. Available from: http://www.sciencedirect.com/science/article/pii/S0022283602013840 
8. Chea E, Livesay DR. How accurate and statistically robust are catalytic site predictions based on closeness centrality? BMC Bioinformatics. 2007;8:153.

9. Atilgan AR, Akan P, Baysal C. Small-world communication of residues and significance for protein dynamics. Biophys J [Internet]. 2004;86(1 Pt 1):85-91. Available from: http://dx.doi.org/10.1016/S0006-3495(04)74086-2

10. Vendruscolo M, Dokholyan N V., Paci E, Karplus M. Small-world view of the amino acids that play a key role in protein folding. Phys Rev E Stat Nonlin Soft Matter Phys. 2002;65(6):4.

12. Negre CFA, Morzan UN, Hendrickson HP, Pal R, Lisi GP, Loria JP, et al. Eigenvector centrality for characterization of protein allosteric pathways. Proc Natl Acad Sci [Internet]. 2018 Dec 26;115(52):E12201 LP-E12208. Available from:

14. Daily MD, Gray JJ. Allosteric communication occurs via networks of tertiary and quaternary motions in proteins. PLoS Comput Biol. 2009;5(2).

410 15. Pintar S, Borišek J, Usenik A, Perdih A, Turk D. Domain sliding of two Staphylococcus 411 aureus $\mathrm{N}$-acetylglucosaminidases enables their substrate-binding prior to its catalysis. 412 Commun Biol. 2020;3(1):1-9.

413 16. Pražnikar J, Tomić M, Turk D. Validation and quality assessment of macromolecular $414 \quad$ structures using complex network analysis. Sci Rep. 2019 Dec 1;9(1).

415 17. Chatterjee S, Bhattacharyya M, Vishveshwara S. Network properties of protein-decoy structures. J Biomol Struct Dyn [Internet]. 2012;29(6):1110-26. Available from: 
https://doi.org/10.1080/07391102.2011.672625

418 18. Chatterjee S, Ghosh S, Vishveshwara S. Network properties of decoys and CASP predicted models: A comparison with native protein structures. Mol Biosyst. 2013;9(7):1774-88.

19. Kleywegt GJ. Validation of protein crystal structures. Acta Crystallogr Sect D Biol Crystallogr. 2000;56(3):249-65.

20. Read RJ, Adams PD, Arendall WB, Brunger AT, Emsley P, Joosten RP, et al. A new generation of crystallographic validation tools for the Protein Data Bank. Structure. 2011;19(10):1395-412.

21. Wlodawer A, Minor W, Dauter Z, Jaskolski M. Protein crystallography for noncrystallographers, or how to get the best (but not more) from published macromolecular structures. FEBS J. 2008;275(1):1-21.

22. Benkert P, Biasini M, Schwede T. Toward the estimation of the absolute quality of individual protein structure models. Bioinformatics. 2011;27(3):343-50.

23. Lobanov MY, Bogatyreva NS, Galzitskaya O V. Radius of gyration as an indicator of protein structure compactness. Mol Biol. 2008;42(4):623-8.

24. Enright MB, Leitner DM. Mass fractal dimension and the compactness of proteins. Phys Rev E - Stat Nonlinear, Soft Matter Phys. 2005;71(1):1-9.

25. Hong L, Lei J. Scaling law for the radius of gyration of proteins and its dependence on hydrophobicity. J Polym Sci Part B Polym Phys [Internet]. 2009;47(2):207-14. Available from: https://onlinelibrary.wiley.com/doi/abs/10.1002/polb.21634

26. Flory PJ. The Configuration of Real Polymer Chains. J Chem Phys [Internet]. 1949;17(3):303-10. Available from: https://doi.org/10.1063/1.1747243

439 27. Tanner JJ. Empirical power laws for the radii of gyration of protein oligomers. Acta Crystallogr Sect D Struct Biol. 2016;72:1119-29.

441 28. Wang G, Dunbrack Jr RL. PISCES: a protein sequence culling server. Bioinformatics [Internet]. 2003 Aug 12;19(12):1589-91. Available from: 
https://doi.org/10.1093/bioinformatics/btg224

29. Grant BJ, Rodrigues APC, ElSawy KM, McCammon JA, Caves LSD. Bio3d: An R package for the comparative analysis of protein structures. Bioinformatics. 2006;22(21):2695-6.

30. Kabsch W, Sander C. Dictionary of protein secondary structure: pattern recognition of hydrogen-bonded and geometrical features. Biopolymers. 1983 Dec;22(12):2577-637.

31. Touw WG, Baakman C, Black J, Te Beek TAH, Krieger E, Joosten RP, et al. A series of PDB-related databanks for everyday needs. Nucleic Acids Res. 2015;43(D1):D364-8.

32. Tien MZ, Meyer AG, Sydykova DK, Spielman SJ, Wilke CO. Maximum allowed solvent accessibilites of residues in proteins. PLoS One. 2013;8(11).

33. Pražnikar J, Tomić M, Turk D. Validation and quality assessment of macromolecular structures using complex network analysis. Sci Rep [Internet]. 2019;9(1):1678. Available from: https://doi.org/10.1038/s41598-019-38658-9

34. Hofmann H, Soranno A, Borgia A, Gast K, Nettels D, Schuler B. Polymer scaling laws of unfolded and intrinsically disordered proteins quantified with single-molecule spectroscopy. Proc Natl Acad Sci U S A. 2012;109(40):16155-60.

35. Kohn JE, Millett IS, Jacob J, Zagrovic B, Dillon TM, Cingel N, et al. Random-coil behavior

36. Wilkins DK, Grimshaw SB, Receveur V, Dobson CM, Jones JA, Smith LJ. Hydrodynamic Radii of Native and Denatured Proteins Measured by Pulse Field Gradient NMR Techniques. Biochemistry [Internet]. 1999 Dec 1;38(50):16424-31. Available from:

465 37. Dima RI, Thirumalai D. Asymmetry in the Shapes of Folded and Denatured States of Proteins. J Phys Chem B [Internet]. 2004 May 1;108(21):6564-70. Available from: https://doi.org/10.1021/jp037128y

38. Gong H, Fleming PJ, Rose GD. Building native protein conformation from highly 
approximate backbone torsion angles. Proc Natl Acad Sci U S A [Internet]. 2005 Nov 8;102(45):16227 LP - 16232. Available from: http://www.pnas.org/content/102/45/16227.abstract

39. Hinsen K, Hu S, Kneller GR, Niemi AJ. A comparison of reduced coordinate sets for describing protein structure. J Chem Phys [Internet]. 2013;139(12):124115. Available from: https://doi.org/10.1063/1.4821598

40. Miller S, Janin J, Lesk AM, Chothia C. Interior and surface of monomeric proteins. J Mol Biol. 1987 Aug;196(3):641-56.

41. Marsh JA. Buried and accessible surface area control intrinsic protein flexibility. J Mol Biol [Internet]. 2013;425(17):3250-63. Available from: http://dx.doi.org/10.1016/j.jmb.2013.06.019

42. Klavzar S, Narayankar KP, Walikar HB, Lokesh SB. Almost-Peripheral Graphs. Taiwan J Math. 2014;18:463-71.

43. Klavžar S, Narayankar KP, Walikar HB. Almost self-centered graphs. Acta Math Sin Eng1 Ser [Internet]. 2011;27(12):2343-50. Available from: https://doi.org/10.1007/s10114-0119628-3

487 The data is freely available on github at https://github.com/jure-praznikar/Scaling-laws. 


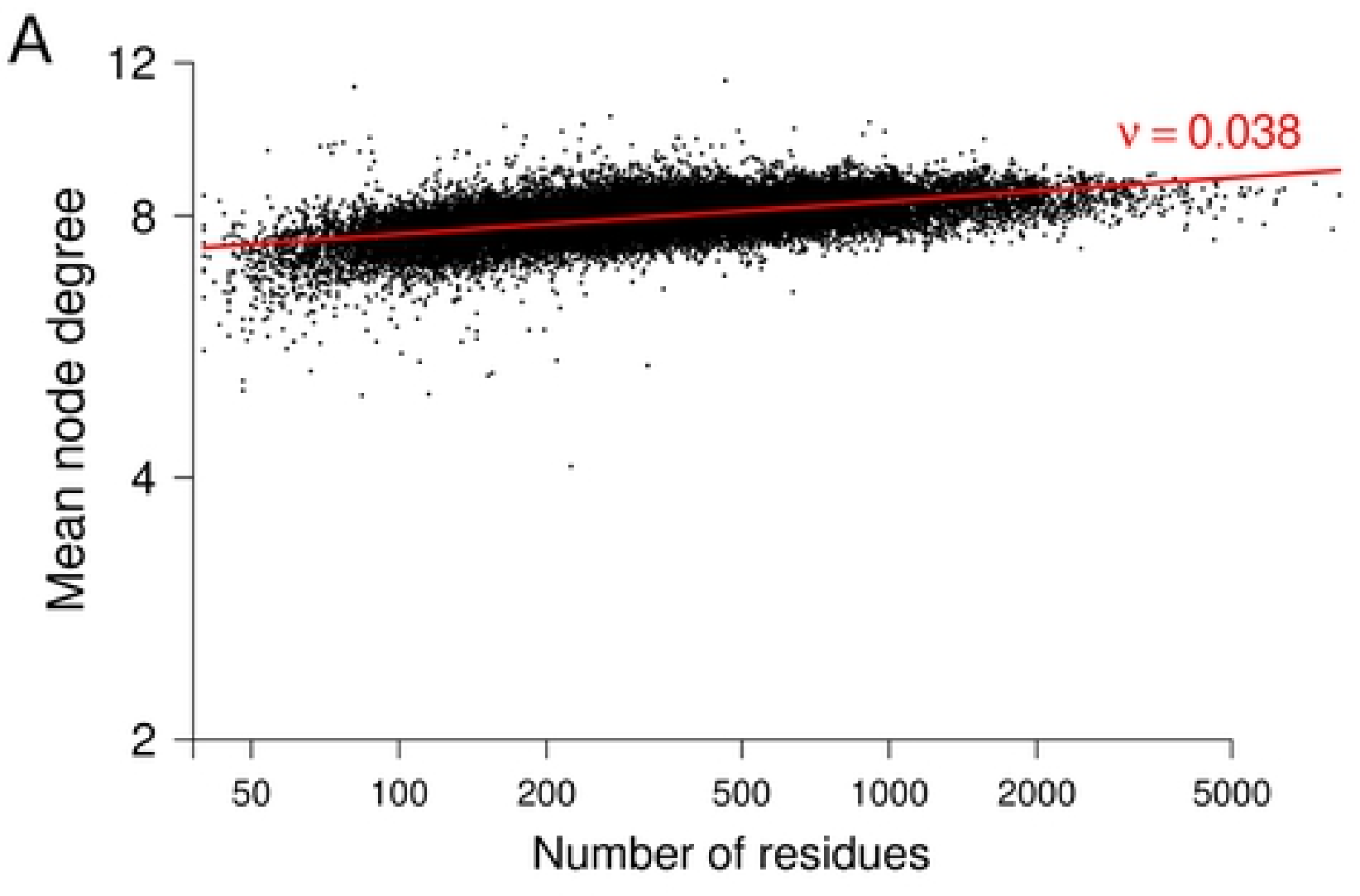

B

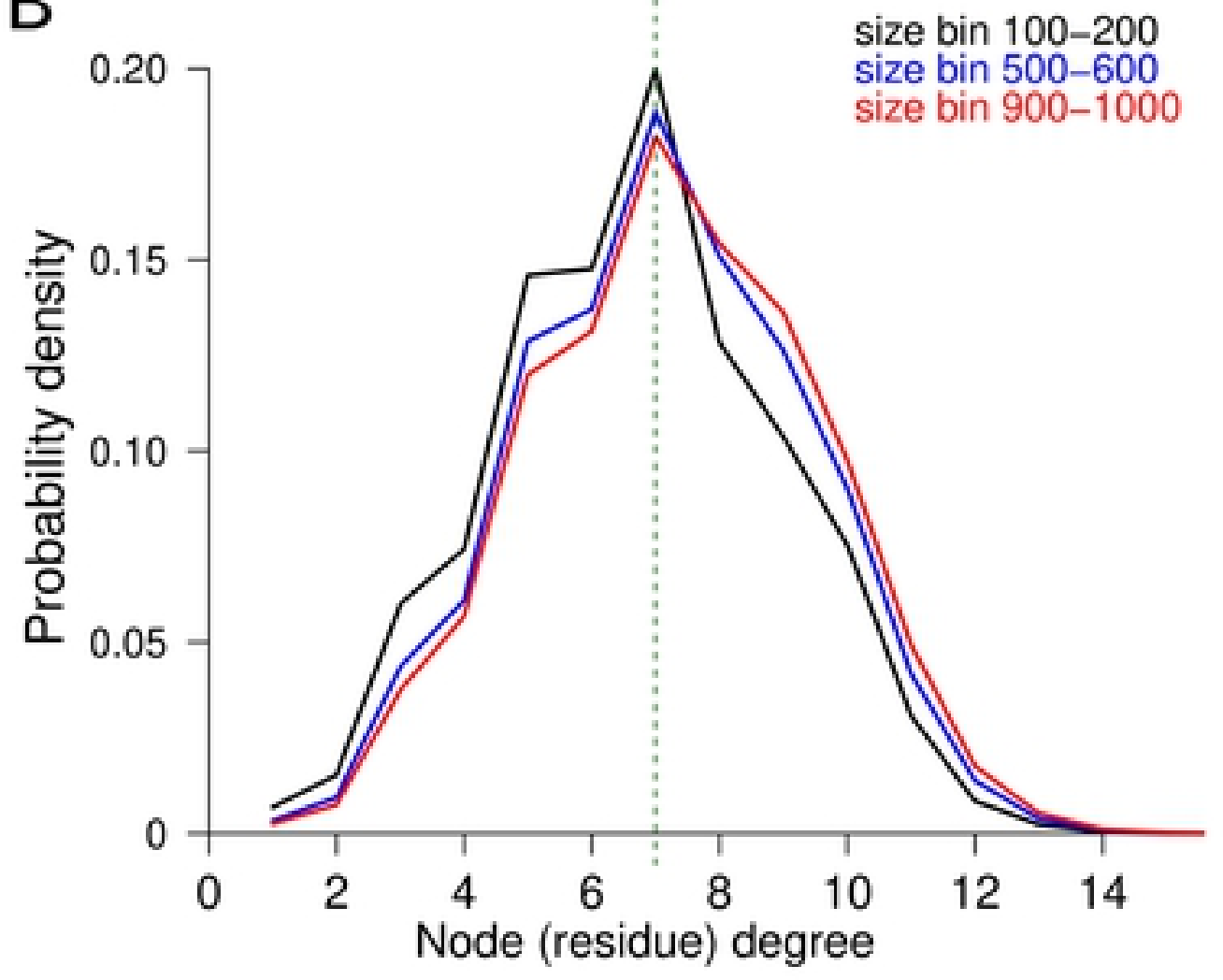

Figure 1 


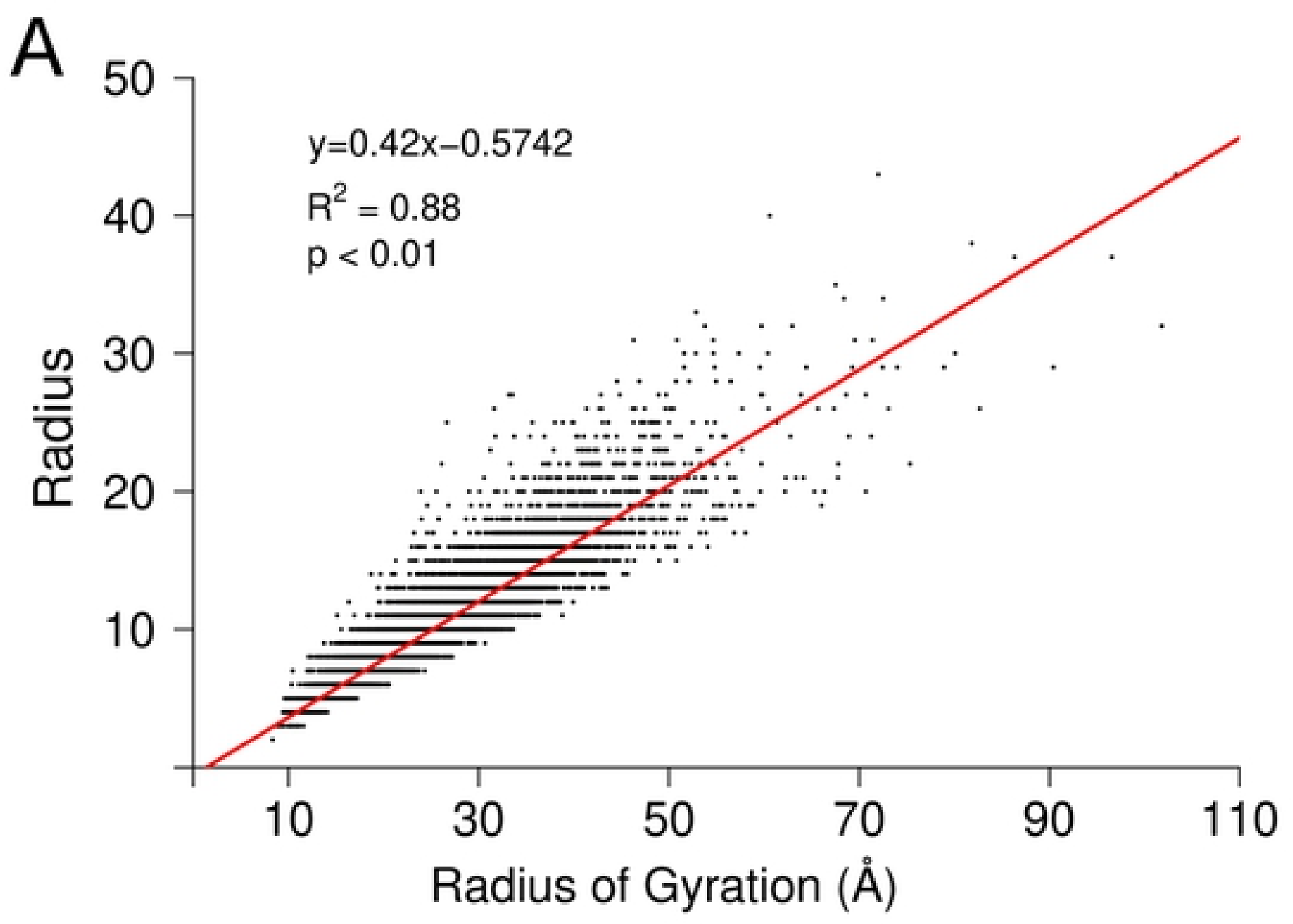

B bioRxiv preprint doi: https://doi.org/10.1101/2020.08.11.246041; this version posted August 11, 2020. The copyright holder for this preprint

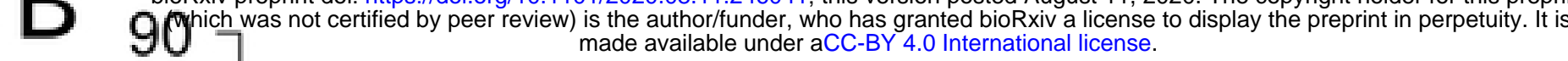

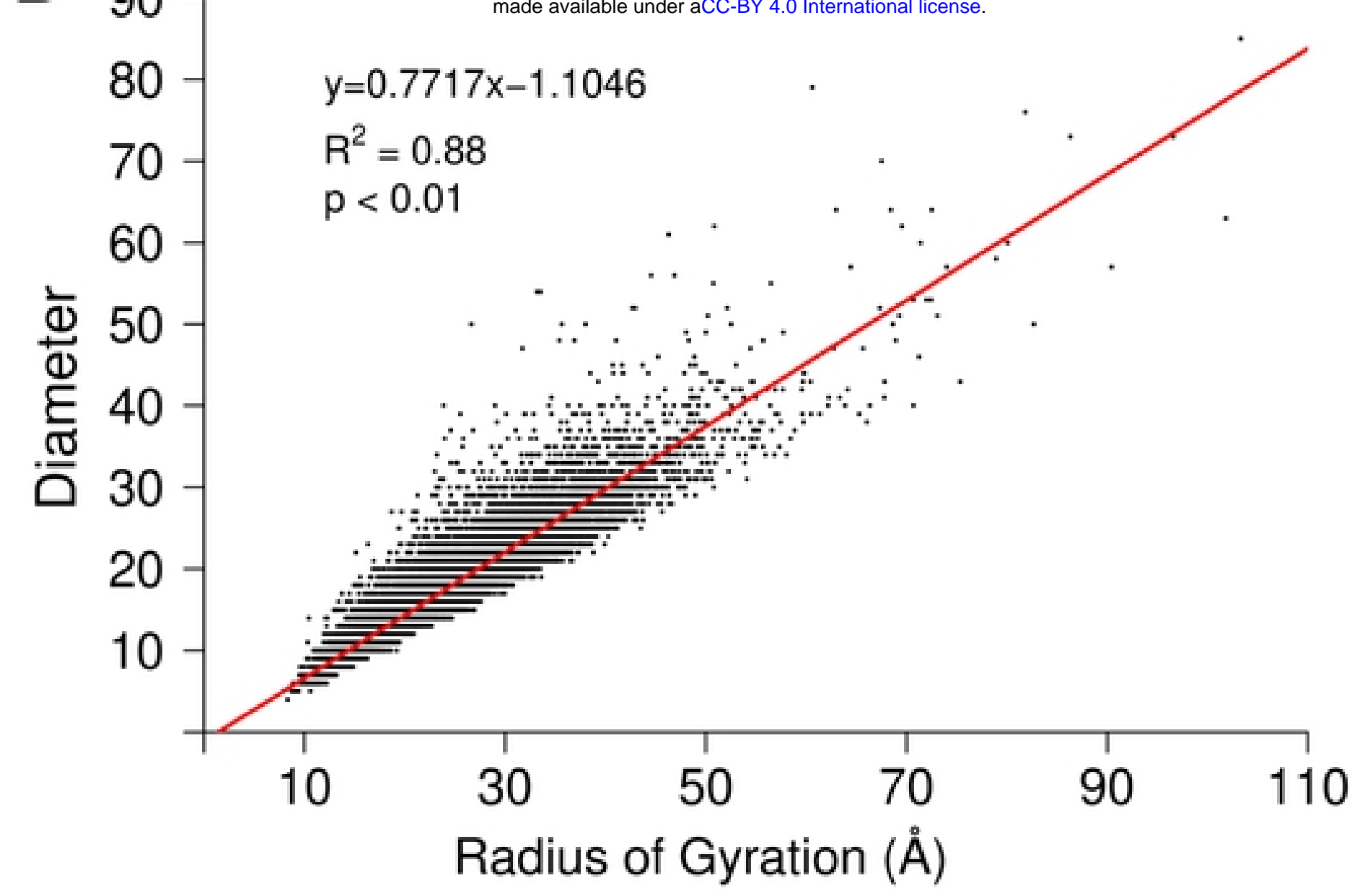

0

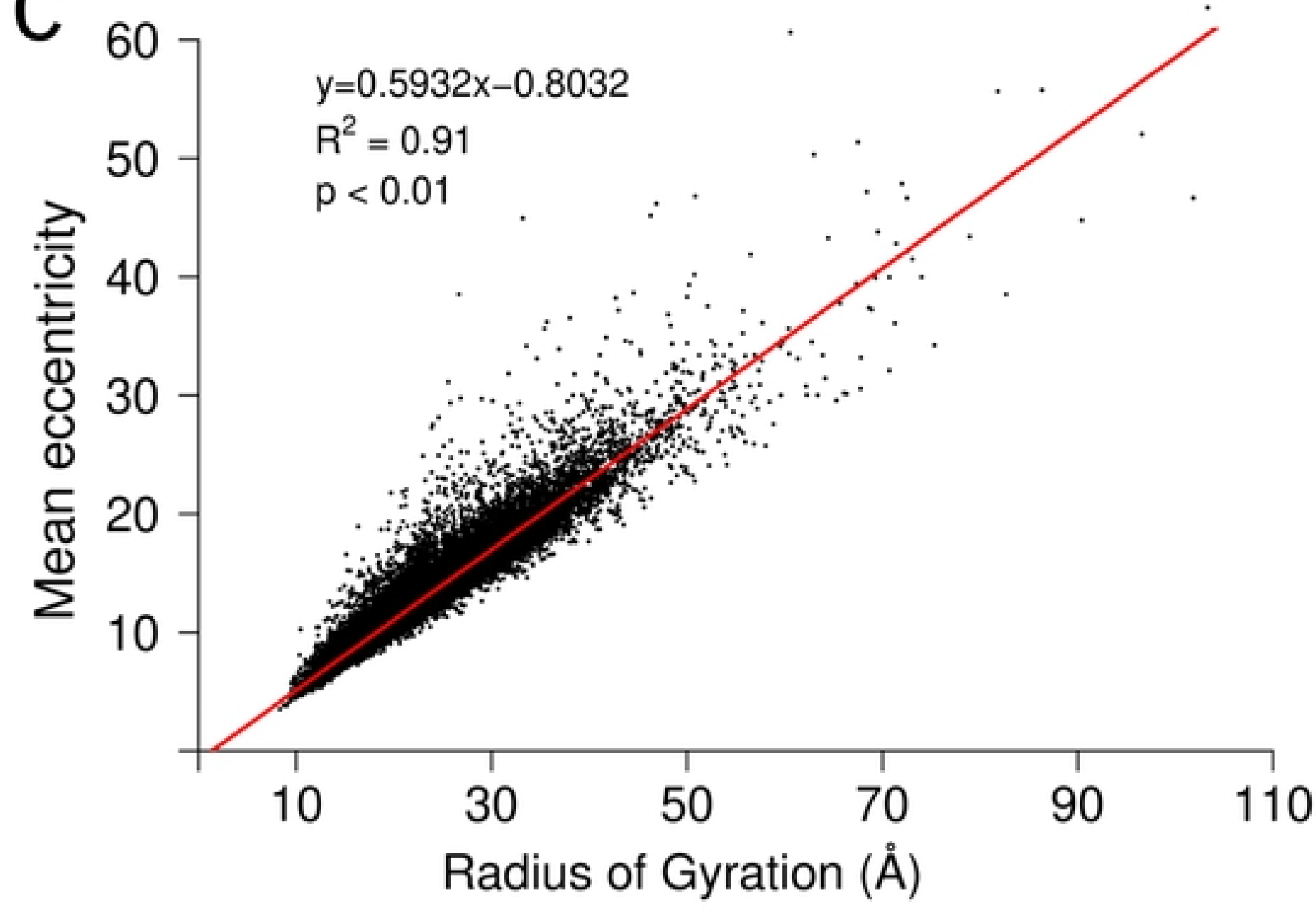

Figure 3 


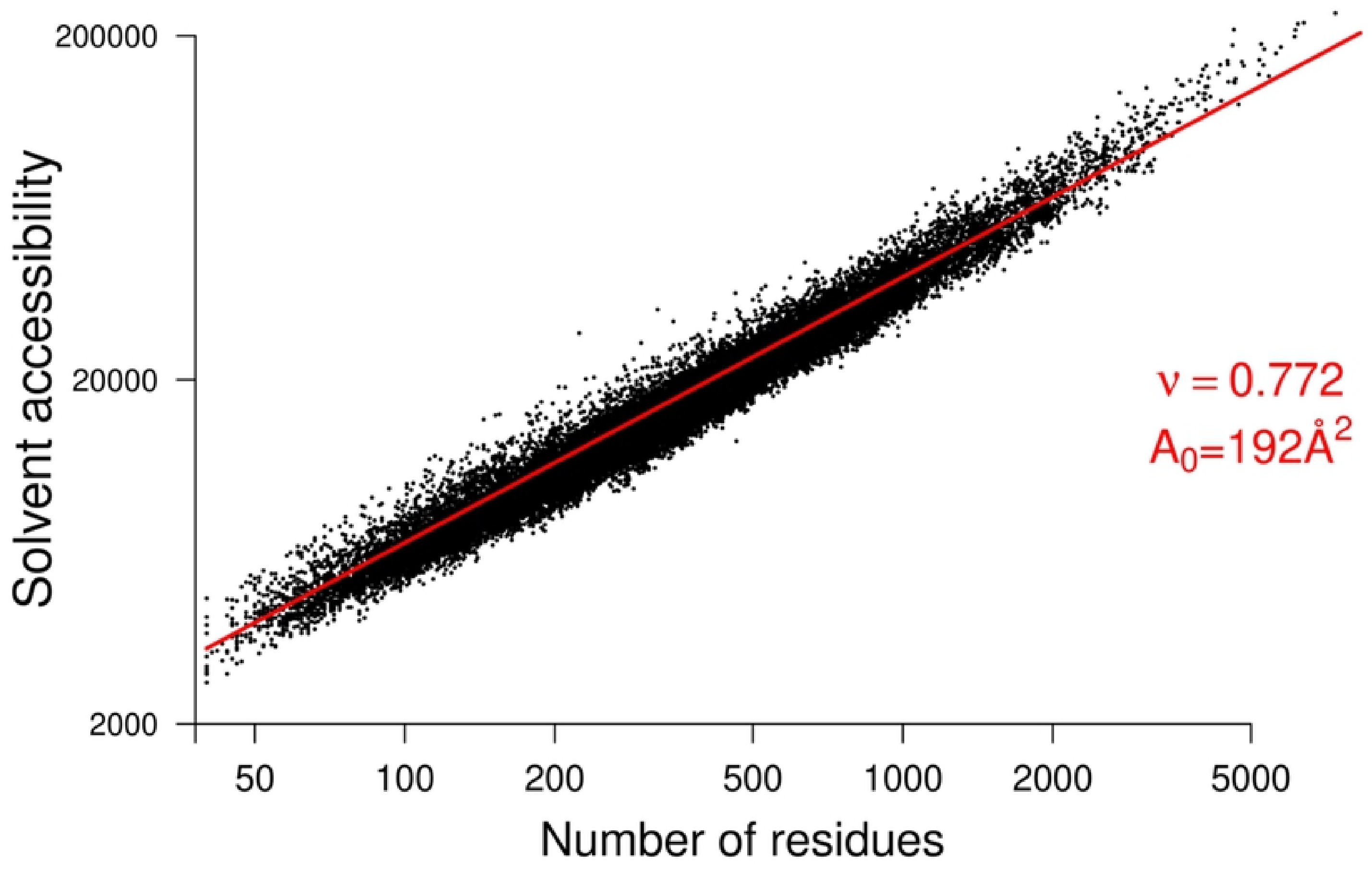

Figure 4 


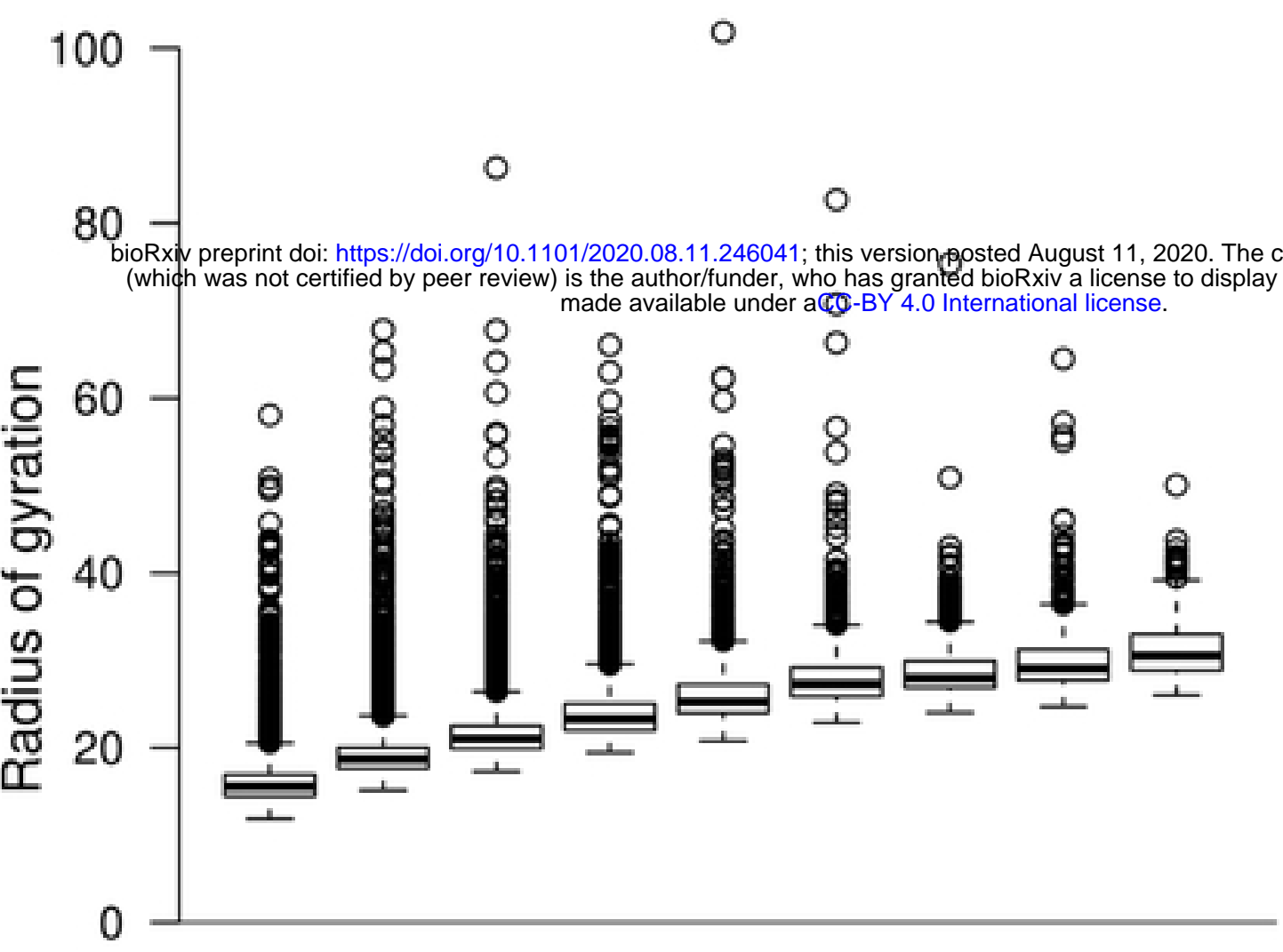

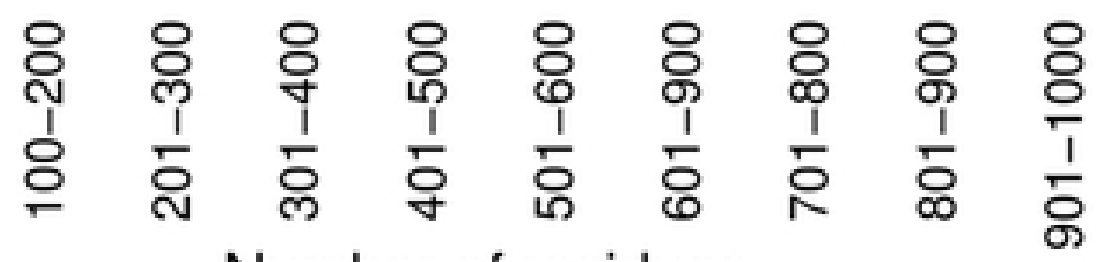
Number of residues
C

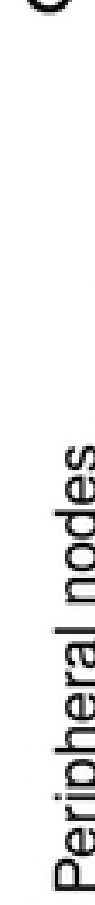

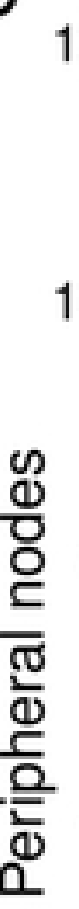

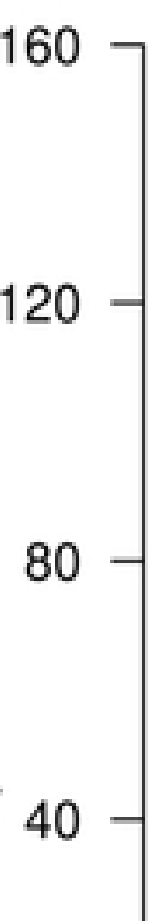

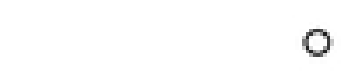

$$
8 \circ
$$

$$
\text { O }
$$

$\circ$

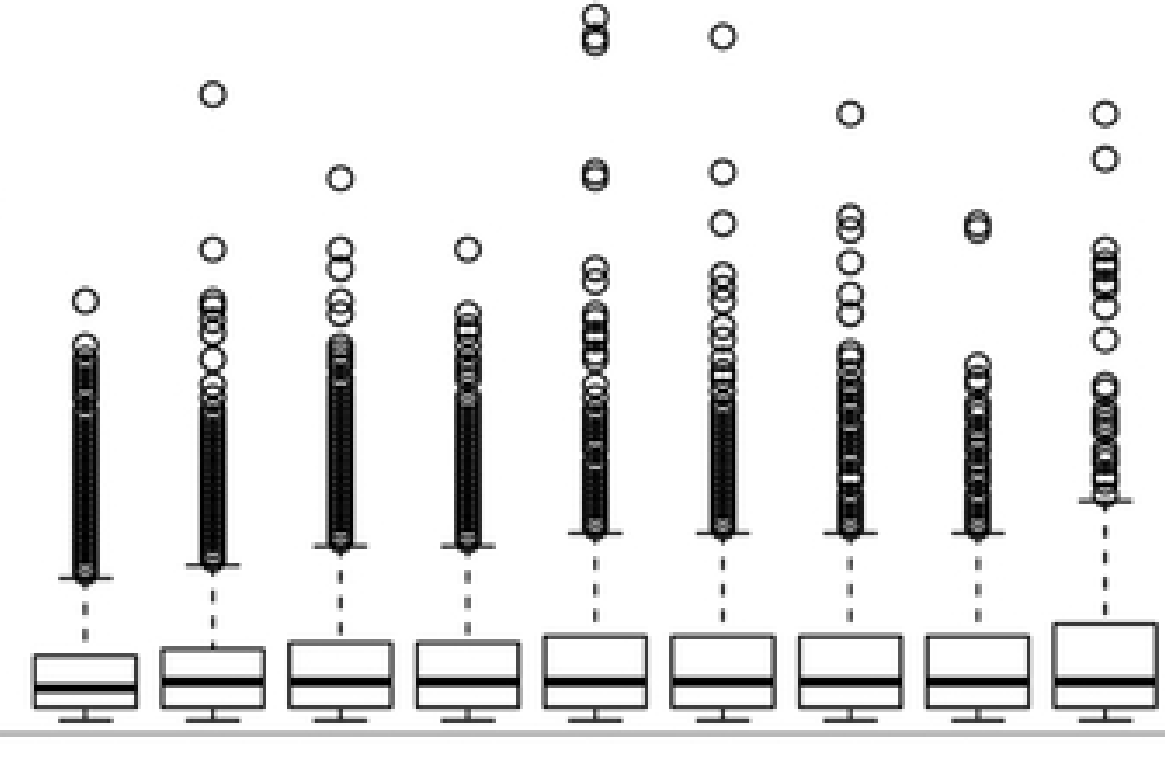

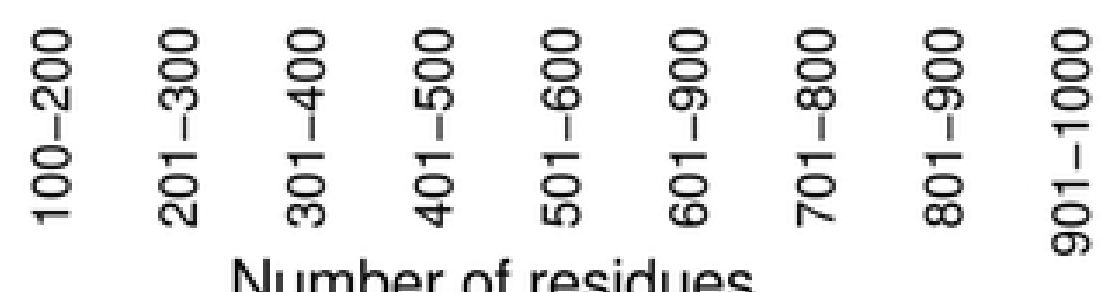

D
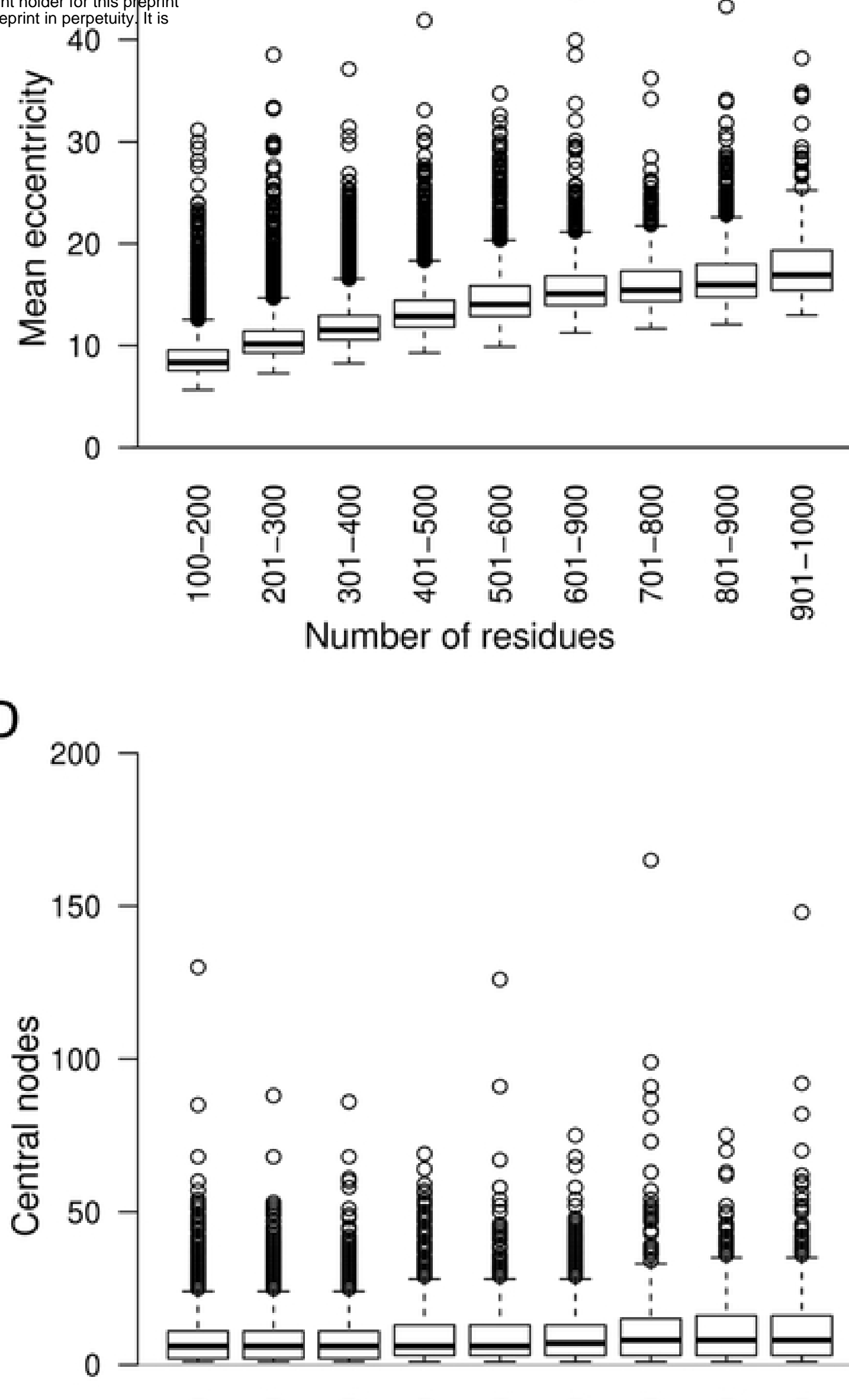

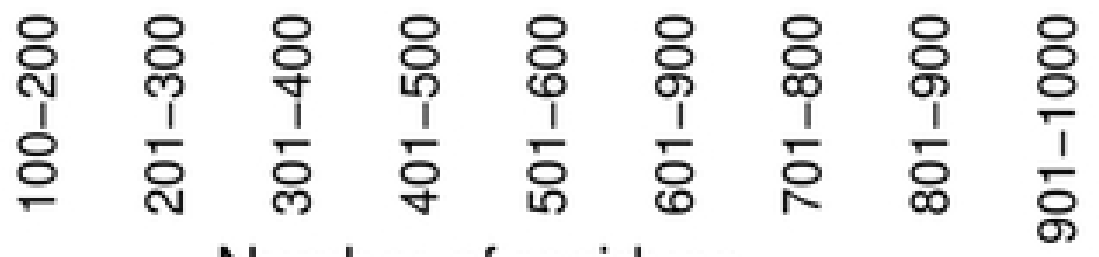
Number of residues

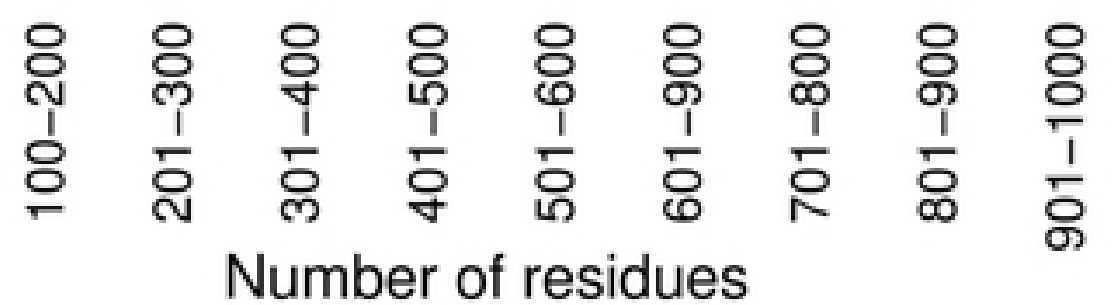

Figure 6 


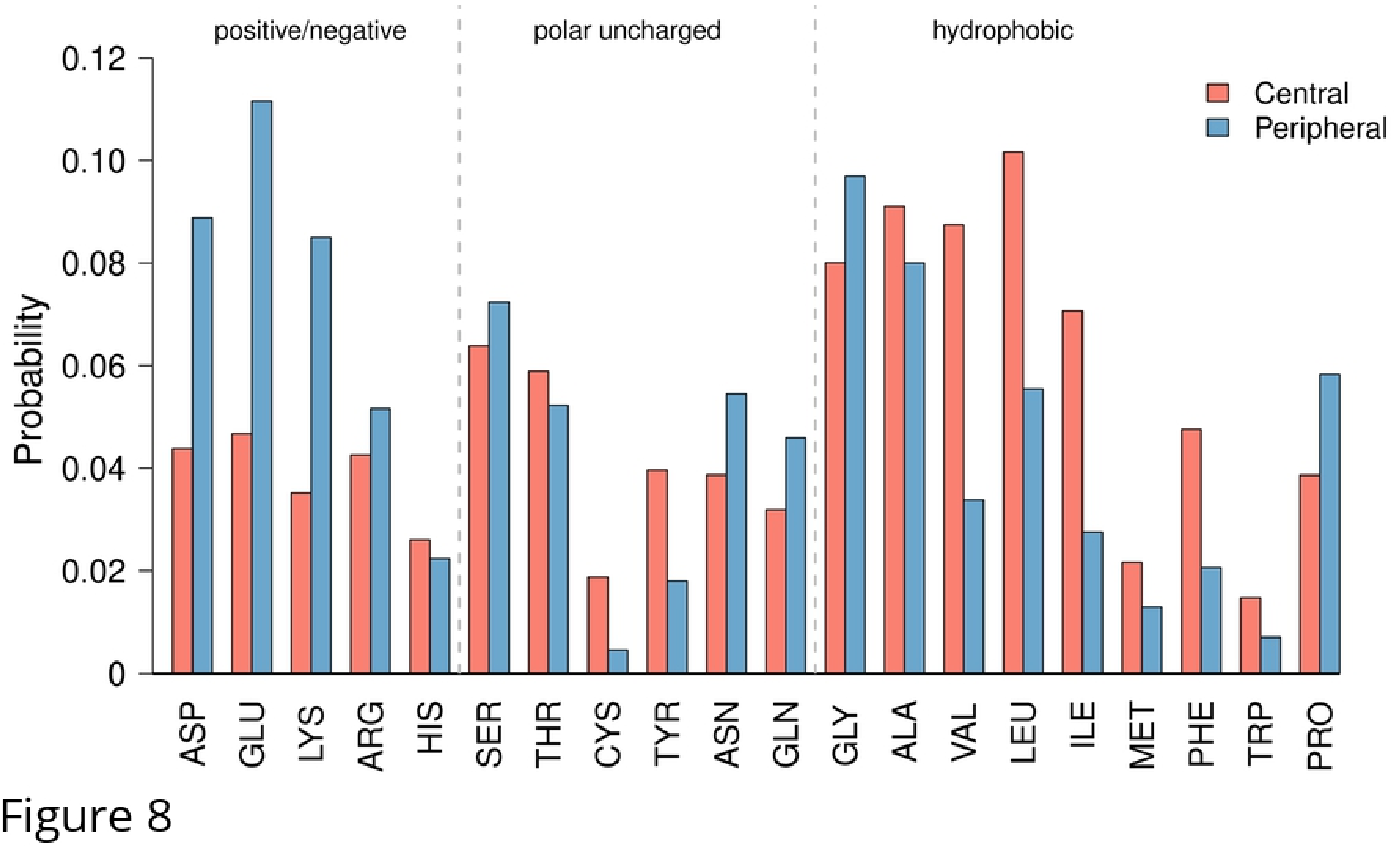

